\title{
Nucleus accumbens activation is linked to salience in social decision making
}

\author{
Stephanie N. L. Schmidt ${ }^{1,2} \cdot$ Sabrina C. Fenske ${ }^{1} \cdot$ Peter Kirsch $^{1} \cdot$ Daniela Mier $^{1,2}$ (D
}

\begin{abstract}
Aberrant salience may explain hasty decision making and psychotic symptoms in schizophrenia. In healthy individuals, final decisions in probabilistic reasoning tasks are related to Nucleus accumbens (Nacc) activation. However, research investigating the Nacc in social decision making is missing. Our study aimed at investigating the role of the Nacc for social decision making and its link to (aberrant) salience attribution. 47 healthy individuals completed a novel social jumping-to-conclusion (JTC) fMRI-paradigm, showing morphed faces simultaneously expressing fear and happiness. Participants decided on the 'current' emotion after each picture, and on the 'general' emotion of series of faces. Nacc activation was stronger during final decisions than in previous trials without a decision, particularly in fear rather than happiness series. A JTC-bias was associated with higher Nacc activation for last fearful, but not last happy faces. Apparently, mechanisms underlying probabilistic reasoning are also relevant for social decision making. The pattern of Nacc activation suggests salience, not reward, drives the final decision. Based on these findings, we hypothesize that aberrant salience might also explain social-cognitive deficits in schizophrenia.
\end{abstract}

Keywords Emotion recognition $\cdot$ Decision making $\cdot$ Jumping-to-conclusion bias $\cdot$ Aberrant salience $\cdot$ Schizophrenia $\cdot$ Nucleus accumbens

\section{Introduction}

Daily, we are faced with the task to recognize other people's emotions. Whereas sometimes, the emotion is very clear and easy to recognize, at other times the facial expression is more subtle or ambiguous, requiring an active decision about the perceived emotion. While this can present a challenge even for people without mental disorders, it is especially difficult for patients with schizophrenia who have impairments in emotion recognition [1,2] and decision making $[3,4]$. Based on the dopamine hypothesis of schizophrenia [5], we hypothesize that decision making for emotions can be disturbed by aberrant Nucleus accumbens (Nacc) activity. To test this assumption we developed a new experimental

Daniela Mier

daniela.mier@uni-konstanz.de

1 Department of Clinical Psychology, Central Institute of Mental Health, Medical Faculty Mannheim/University of Heidelberg, J5 68159 Mannheim, Germany

2 Present Address: Department of Psychology, University of Konstanz, Konstanz, Germany paradigm that combines emotion recognition with decision making and applied it to a group of healthy participants.

Nacc and the fronto-parietal network, including parietal cortex and dorsolateral prefrontal cortex (DLPFC) are key regions for decision making [6-9]. Final decisions during probabilistic reasoning tasks are related to increased activation in ventral tegmental area (VTA) and Nacc in healthy participants, whereas schizophrenia patients (SZ) have reduced activation in these areas [10]. The Nacc, which is a part of the ventral striatum, has a high density of dopamine receptors and is a central region for motivation, reward and pleasure [11] with a major role for reward anticipation $[11,12]$ and salience attribution [13-15]. Dysfunction of the dopaminergic system appears to build the foundation of deficits characteristic for SZ which led to the "dopamine hypothesis of schizophrenia" [5]. In particular chaotic dopaminergic signaling in the Nacc has been proposed to be causal to the aberrant salience attribution in SZ, and hypersalience, i.e., enhanced salience attribution to seemingly neutral objects, has been assumed to cause delusions [15].

Hasty decision making is known to occur in schizophrenia [4]. A recent meta-analysis confirmed that people with 
psychosis decide based on significantly less evidence than healthy, as well as clinical populations without psychosis. Importantly, it was shown that a JTC-bias is specifically linked to delusions [16]. In decision making, hypersalience may put too much weight on current information, leading to insufficient data gathering and thus hasty decisions, also called jumping-to-conclusion (JTC) bias [17]. While hypersalience may cause the JTC-bias in (non-social) decision making tasks, it may lead to wrong attributions of emotions and mental states to others in emotion recognition [18], which again may support the emergence of delusions [19]. Interestingly, there is evidence that the deficit in emotion recognition in SZ is most pronounced for ambiguous or neutral facial expressions [2, 20]. Ambiguous facial expressions are defined by the existence of more than one emotion, making a decision process for emotion recognition necessary. Since neutral facial expressions are defined by the absence of any emotion, false emotion recognition always implies the false perception of an emotion; i.e., a false-positive decision for the existence of an emotion. Thus, hasty decision making and hypersalience might have a special role for biases in the recognition of ambiguous and neutral facial expressions, possibly suggesting an interaction of disturbed decision making and emotion recognition.

Aberrations in Nacc activity in SZ have not only been shown for decision making [21], but present a stable finding for reward anticipation in SZ [22, 23]. Thus, it is an interesting question whether reward or salience is the mechanism causing enhanced Nacc activity during final decision making, and consequently aberrant Nacc activity in SZ. Esslinger and colleagues found no differences in Nacc activation between rewarded and unrewarded final decisions, and concluded that Nacc activity reflects salience rather than the rewarding impact of the last stimulus [14]. However, more studies directly investigating factors influencing Nacc activity during the final decision are necessary. Additionally, our knowledge on Nacc activation in decision making is based on "non-social" decision making tasks, leaving the question of the role of the Nacc for decision making during emotion recognition, i.e., in social decision making. Usually, emotion recognition has been associated with activation in the amygdala [24], best known for its role in fear processing, including recognition of fearful faces [25-27]. Moreover, the amygdala can reflect the salience of facial expressions [28]. To a lesser extent than in fear, the amygdala is also activated in other negative and even positive emotions, including happiness [29]. The anticipation of reward is linked to Nacc activation, for both monetary and social reinforcers [30, 31]. Watching happy and attractive faces is considered rewarding, and activates the Nacc [29, 32, 33]. Facial expressions with a negative valence (e.g., fear, anger), however, are not considered rewarding, but instead indicating threat and thus aversive conditions (e.g., 34, 35). They are detected more quickly and accurately than happy faces in face-in-the-crowd tasks, suggesting higher salience for negative than positive facial expressions [36, 37]. Importantly, the Nacc maintains connections to the amygdala [38], suggesting interactions of amygdala and Nacc that might also be relevant for emotion recognition. Thus, both fear and happiness can be salient, but evidence suggests that fear is more salient than happiness and usually fear is not rewarding. We apply the knowledge of tasks using emotional stimuli to our social decision making task with the following logic: If increased Nacc activity during final decision making is associated with reward rather than salience, we expect it to be more prominent in the case of happy than fearful final stimuli.

Taken together, findings on final decision making in schizophrenia suggest reduced activation in the Nacc [21], while based on the dopamine hypothesis [39] enhanced Nacc activation would be predicted. Further, until now it is not clear whether this reduced Nacc activation during final decision making in SZ is based on aberrant salience, or aberrant reward anticipation. Since patients with SZ show impaired decision making [16], as well as deficits in social cognition [40], investigating the interaction of these processes seems highly warranted. In our fMRI study, we include healthy participants to investigate whether findings from non-social JTC-tasks can be replicated in a novel social JTC paradigm, which requires emotion recognition in mixed (morphed) facial expressions. We hypothesize that probabilistic decision making for emotion recognition leads to activation in the fronto-parietal network and that the final decision of a probabilistic reasoning process is linked to Nacc activation. Using faces showing fear and happiness in varying degrees, we aim to explore whether salience or reward is linked to Nacc activity during final decision making. If Nacc activation is related to reward rather than salience, Nacc activation should be stronger for happiness than fear. To get first evidence of the link between schizophrenia pathology and activation in the Nacc during final decision making in this social decision making task, we (a) assess personality traits (schizotypy), and measures of social functioning (social network size and diversity), and (b) compare participants according to their decision behavior.

\section{Materials and methods}

\section{Participants}

47 healthy, right-handed Caucasian individuals with a general qualification for university entrance [29 women, 18 men; mean age 23.4 years ( \pm 3.6 ), range $18-33$ years] underwent functional magnetic resonance imaging in a Siemens Magnetom Trio 3T (Central Institute of Mental Health in Mannheim, Germany). Exclusion criteria were all assessed 
based on self-report and comprise a history of neurologic or psychiatric disease and presence of other diseases which require constant medication, as well as the general exclusion criteria for fMRI.

\section{Study procedure}

The experiment was conducted as part of a study that was approved by the ethics committee of the University of Heidelberg and in agreement with the Declaration of Helsinki. Participants were informed about study aims and procedures, signed written informed consent, received oral and written instruction on the paradigm, and completed a battery of questionnaires. Before the MR session, each participant practiced the paradigm until it was familiar and clear. Practice runs entailed the same identities as those used in the experiment. In contrast to experimental stimuli, which were based on fearful and happy facial expressions, practice stimuli were morphs between angry and happy, or between disgusted and happy faces. In the MR scanner, participants held a Current Designs 4-button diamond device in their right hand and watched the paradigm via video goggles. Prior to the experimental task and measurement, an MPRage anatomical measurement was performed, during which a nature movie was shown, so participants could get acquainted to the MR environment. Participants were reimbursed with $15 €$.

\section{Experimental design}

In the style of the classical beads task [41] and the modified JTC-task [14, 42], we developed a social JTC paradigm ("Jemo"), which combines recognizing emotions in emotionally ambiguous faces with decision making. The happy and fearful facial expressions of six Caucasians (three women, three men) of the NimStim Face Stimulus Set (http://www. macbrain.org/resources.htm, [43]) were selected, and for each individual, the happy and fearful face were morphed in 5\% steps, ranging from $0 \%$ (0\% fearful, $100 \%$ happy) to $100 \%$ (100\% fearful, $0 \%$ happy). The morphed pictures were taken from Matzke and colleagues [44]. In a pilot study, 25 healthy students judged which of the two emotions was predominant in each picture. Based on these ratings, we determined 7 morphs per stimulus person with the fourth morph being close to a 50/50 rating across participants, and the other 6 morphs having an increasing percentage of fear (3) or happiness (3) (see Fig. 1 for examples).

In the Jemo paradigm, the most ambiguous fourth morph is presented as the first stimulus in a series of maximum 5 pictures. Each of the following morphs is less ambiguous, either more happy or more fearful. Every series of five stimuli has one incongruent stimulus, in which the recessive emotion prevails. On average, the incongruent morph consists of 77\% (range 61-92\%) of the recessive emotion. The incongruent trial appears in second, third, or fourth position. The task of the participants is to identify (a) the emotion of each stimulus (referred to as current emotion), and (b) the predominant emotion in a series (referred to as general emotion) as soon as possible. If the participant correctly identifies the incongruent stimulus, the current emotion is correct. However, if the participant wrongly determines the incongruent stimulus to reflect the prevailing emotion within the series, this is considered an incorrect decision on the general emotion. Each picture is presented for $2 \mathrm{~s}$, after which participants have $2 \mathrm{~s}$ to decide on the emotion displayed in this picture (current emotion), indicate their certainty about the decision within $4 \mathrm{~s}$, and decide within $2.5 \mathrm{~s}$ whether they want to see another picture or already know the general emotion; in the latter case, they subsequently have $2 \mathrm{~s}$ to decide on the general emotion. Stimuli within a trial are presented with a jittered inter-stimulus-interval of $1 \mathrm{~s} \pm 0.5 \mathrm{~s}$, distinct series are separated by a jittered intertrial-interval of $2 \mathrm{~s} \pm 1 \mathrm{~s}$. A fixation cross is presented during the inter-stimulus/trial-intervals. There are 24 trials in total (6 identities with 2 emotional directions, all presented twice). Duration of the experiment was dependent on the number of stimuli participants needed for a decision. They were told, however, that the experiment takes around $15 \mathrm{~min}$ and were not aware that taking fewer stimuli to decide would reduce experimental time.

\section{Questionnaires}

Participants completed questionnaires assessing schizotypy (Schizotypal Personality Questionnaire, SPQ [45]) and social network behavior (Social Network Index, SNI [46]). Schizotypy refers to a combination of personality traits that
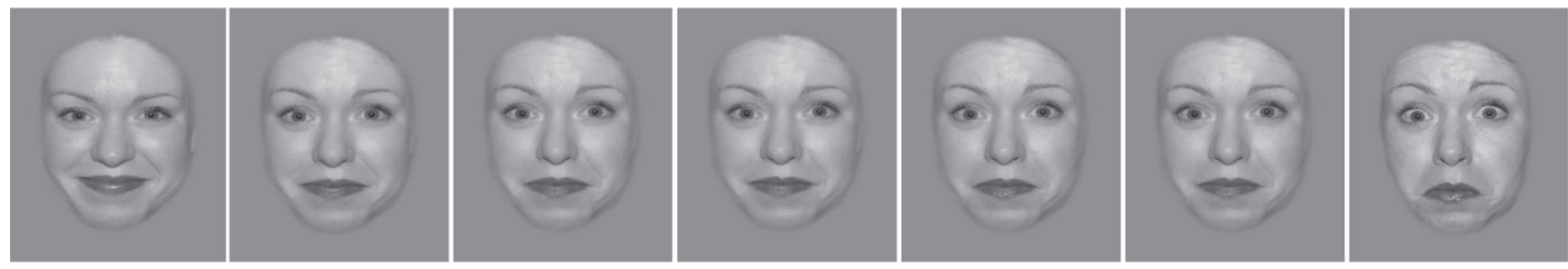

Fig. 1 Example of stimuli: left: most happy picture, middle: 50/50 morph fearful-happy, right: most fearful picture, increments in between 
largely overlap with symptoms of schizophrenia, both behaviorally and neurobiologically [47]. The SPQ consists of 9 subscales, and includes the central aspects of schizoptypy such as constricted affect, unusual perceptual experiences and suspiciousness. The SNI assesses social ties in the private and professional environment, and is evaluated regarding three subscales: (1) Network Diversity, which reflects the number of social roles, in which the individual has regular contact, e.g., parent, child, spouse, employee, neighbor. (2) People, which counts the total number of people an individual is in regular contact with. (3) Roles, which reflects the number of different network domains in which an individual is active, which is based on the number of high-contact people in each network, e.g., family, work, neighbors. Previous studies suggest reduced social networks already in people with subclinical psychotic experiences [48].

\section{fMRI data acquisition and analysis}

fMRI data was acquired using a 12-channel head coil in a $3 \mathrm{~T}$ Siemens Magnetom Trio at the Central Institute of Mental Health in Mannheim, Germany. During the tasks, we used echo-planar imaging with 32 descending $3 \times 3 \times 4 \mathrm{~mm}$ slices including $1 \mathrm{~mm}$ gap, TR $=2000 \mathrm{~ms}, \mathrm{TE}=30 \mathrm{~ms}$, flip angle $=80^{\circ}$, field of view $=192 \mathrm{~mm}$, matrix $=64 \times 64$.

Data were analyzed using SPM8 (http://www.fil.ion.ucl. ac.uk/spm/software/spm8/), with preprocessing including slice time correction, realignment, normalization to MNI space with resampling to $3 \times 3 \times 3 \mathrm{~mm}$ voxels, and spatial smoothing with a $8 \mathrm{~mm}$ full-width half-maximum Gaussian filter. We used a high-pass filter of 512 s. First-level analysis included seven regressors (last fearful face, last happy face, previous fearful faces, previous happy faces, happy block, fearful block, key presses) in a hybrid design modelling tonic (i.e., blocks of probabilistic reasoning) and phasic activity (i.e., events of final decision making and events without a final decision), according to our earlier publications with a non-social decision making paradigm [14, 21]. The purpose of this hybrid design was twofold: (a) it allows analyzing phasic as well as tonic responses occurring in the experiment, and (b) activation revealed with event-modulation is attributable to phasic effects under control of tonic effects, while the opposite is true for block-modulation. The contrasts for probabilistic reasoning were blocks with faces increasing in happiness and blocks with faces increasing in fear (> baseline fixation cross; block modulation). The contrast for final decision making was the difference in activation between the last face and all previous faces (event-modulation). We also analyzed the interaction of brain activation during fearful last versus previous stimuli in comparison to happy last versus previous stimuli (event-modulation).

In second-level random-effects group analyses, we applied $t$ tests to the contrasts of interest. Our regions of interest (ROI) included BA40 and BA7 (parietal cortex), BA46 and BA9 (DLPFC) for probabilistic reasoning, and Nacc and amygdala for final decision making. The masks were taken from the wakeforest university pickatlas (WFU Pickatlas, http://fmri.wfubmc.edu/software/PickAtlas). The Nacc mask was drawn according to an anatomic atlas and has already been successfully applied in our earlier studies with a JTC design [49]. The significance threshold for whole-brain analyses was set to $p<0.05$, corrected for multiple testing using family-wise error (FWE), and a minimal cluster size of $k=5$ voxels. ROI significance was set to $p<0.001$, uncorrected, $k=5$ with $p<0.05$ small volume correction (svc) of the peak voxel. The Nacc mask had a size of 128 voxels on the left, and 93 voxels on the right side.

Questionnaires were analyzed with IBM SPSS Statistics 22 (Chicago, IL, USA). Correlations of brain activation with questionnaires and behavior were calculated based on contrast estimates extracted from the Nacc ROI for the contrast all last faces $>$ all previous faces (to assure the same number of voxels for eigenvariate extraction across participants, no significance threshold was set; i.e., $p=1$ ). In addition, behavioral subgroups of participants were compared with regard to their Nacc contrast estimates. Behavioral data were analyzed by repeated measures ANOVAs and $t$ tests.

\section{Results}

\section{Behavior}

Both, in blocks with increasingly happy and in blocks with increasingly fearful faces, subjects watched on average three pictures [happy: mean $=3.02, \mathrm{SD}=0.94$; fearful: mean $=2.97, \mathrm{SD}=1.01 ; t(46)=1.04, p=0.306$, $d=0.05]$. There was no significant difference in performance between recognizing the current emotion in happy or fearful faces [happy: mean $=62.74 \%, \mathrm{SD}=22.92$; fearful: mean $=64.81 \%, \mathrm{SD}=21.31 ; t(46)=0.37, p=0.71, d=0.09]$. Also, correctness of the decision on the general emotion within a block was not significantly different between the two emotion conditions [happy: mean $=54.34 \%, \mathrm{SD}=30.80$; fearful: mean $=60.72 \%, \mathrm{SD}=30.91 ; t(46)=0.96, p=0.34$, $d=0.21]$. As illustrated in Fig. 2, both accuracy and certainty of decisions increased with the number of stimuli considered. We performed a repeated measures general linear model to test the main effect number of stimuli on accuracy and certainty in fear and happiness blocks. There was a significant effect of stimulus number within a series on correct decisions in happiness blocks: $F(4,116)=32.27$, $p<0.001, \eta_{\mathrm{p}}{ }^{2}=0.53$; on correct decisions in fear blocks: $F(4$, $124)=58.48, p<0.001, \eta_{\mathrm{p}}{ }^{2}=0.65$; on certainty in happiness blocks: $F(2.77,80.39)=55.08, p<0.001, \eta_{\mathrm{p}}{ }^{2}=0.66$; on certainty in fear blocks: $F(3.25,100.69)=21.40, p<0.001$, 
Fig. 2 Mean percentages of correctly recognized emotions, and certainty ratings as bars. Lines indicate standard deviations

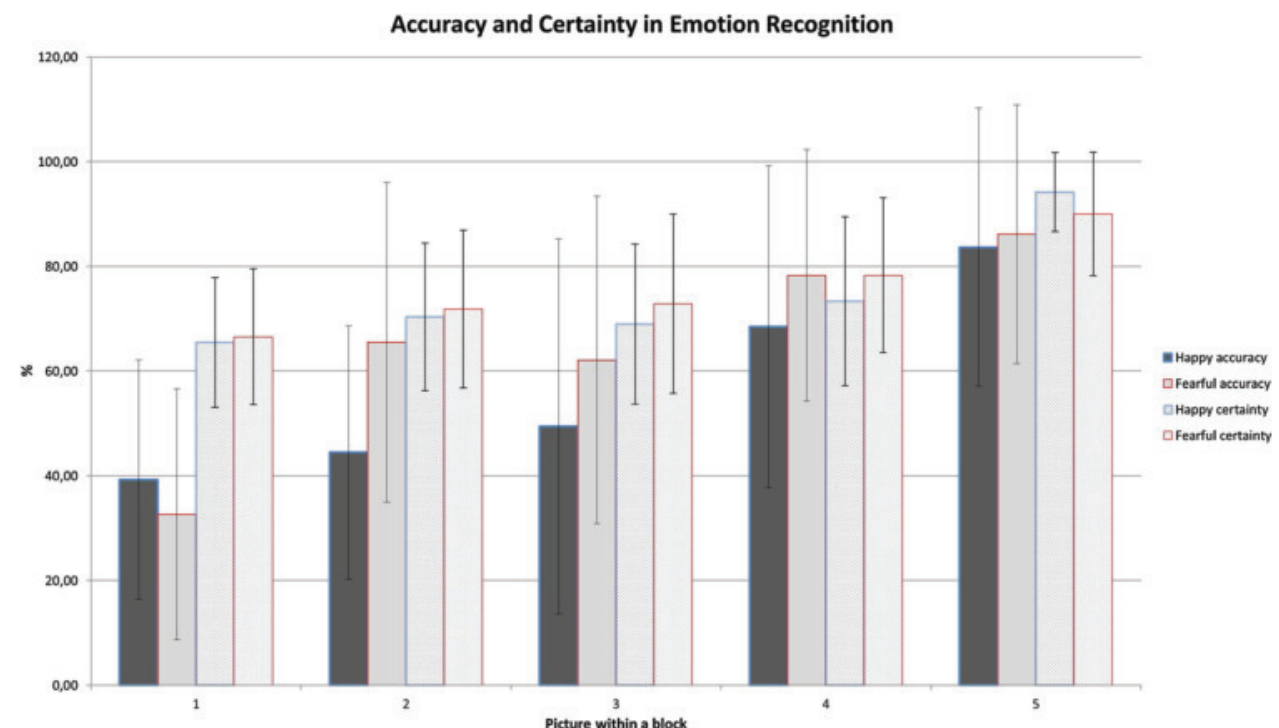

$\eta_{\mathrm{p}}{ }^{2}=0.41$. Reaction times were not significantly different in the happy and fearful series [happy: mean $=733 \mathrm{~ms}$, $\mathrm{SD}=142$. fearful: mean $=724 \mathrm{~ms}, \mathrm{SD}=127 . t(46)=0.68$, $p=0.500, d=0.07]$. Experimental duration varied between participants depending on the number of stimuli they considered, and was on average $12.76 \mathrm{~min}(\mathrm{SD}=3.84)$. The number of draws-to-decision (DTD) correlated significantly with the accuracy of decisions for the current (happiness block: $r=0.512, p<0.001$; fear block: $r=0.312 ; p=0.033$ ) and for the general emotion (happiness block: $r=0.481, p<0.001$; fear block: $r=0.453 ; p=0.0014)$.

Exploratorily, we additionally analyzed whether the incongruent faces within each trial affected the decision process. On average, participants saw incongruent faces in $73.3 \%$ of trials $(\mathrm{SD}=23.4 \mathrm{~s})$. When participants decided on the general emotion immediately after the presentation of an incongruent face, performance was below chance level ( $25.4 \%$ correct responses, $\mathrm{SD}=32.2)$ with happiness as the dominant emotion within a series, and $34.8 \%$ correct responses $(\mathrm{SD}=35.9)$ with fear as the dominant emotion within a series. However, a decision on the general emotion of a series was only made after presentation of an incongruent face in $25.7 \%$ of series $(\mathrm{SD}=14.4)$.

\section{Brain activation}

Whole-brain analyses revealed activation in the visual association cortex, and in parietal (BA7) and frontal (BA6 and BA44) lobe for fear blocks. During the happiness block, there was also enhanced activation in visual association cortex, parietal (BA7) and frontal (BA6) lobe. ROI-analyses confirmed these results from the whole-brain analyses. In both the fear block and the happiness block, there was activity in the DLPFC, and parietal cortex ROIs.

During all last faces compared to all previous faces, activation was increased in the bilateral putamen, with the cluster reaching into Nacc, and the anterior cingulate cortex (ACC; Fig. 3). ROI-analyses confirmed enhanced Nacc activity for the last faces in comparison to all previous faces. This activation pattern was mainly driven by the fearful series comparing last to previous faces, but not by the contrast happy last versus happy previous. The interaction
Fig. 3 Whole-brain activation for all last $>$ all previous faces, $p<0.05$, FWE-corrected, $k=5$, at coordinates: $x, y, z=2,14$, - 11. a Nucleus accumbens, b anterior cingulate cortex
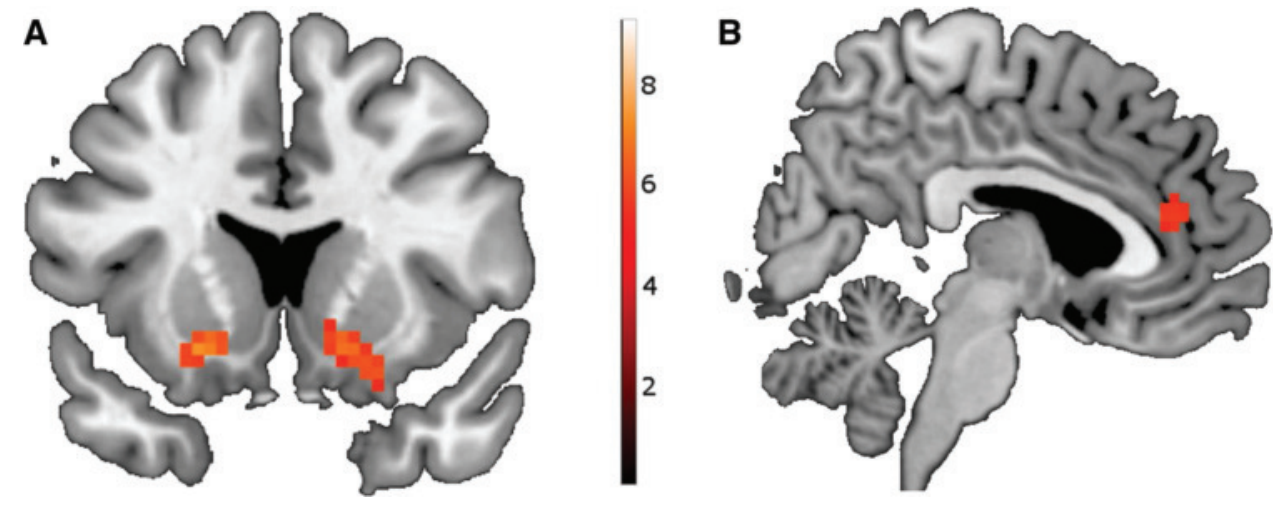
contrast (Fig. 4) comparing the last fear face to all previous fear faces in comparison to the last happy face to all previous happy faces, revealed no significance at the whole brain corrected threshold, but ROI-analyses showed stronger Nacc activation for fearful rather than happy last stimuli. None of the contrasts showed significant activation differences in the amygdala. Results of the whole-brain analyses are presented in Table 1, ROI-analyses in Table 2.

\section{Brain-behavior associations}

Our sample included an extreme group of five participants, who on average looked at less than two faces before deciding on the general emotion $(1.45 \pm 0.37)$ which is considered as JTC-bias $[10,16]$. We compared them to the other 42 participants $[3.18 \pm 0.83$; difference between groups: $t(10.1)=8.30, p<0.001, d=2.70]$. We refer to the group

A

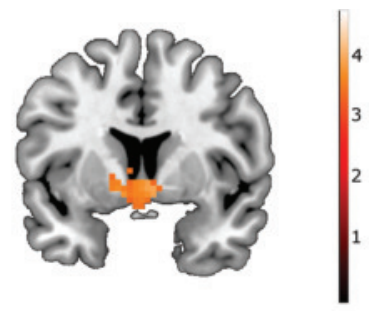

B

Nacc activation during previous and last fearful and happy faces

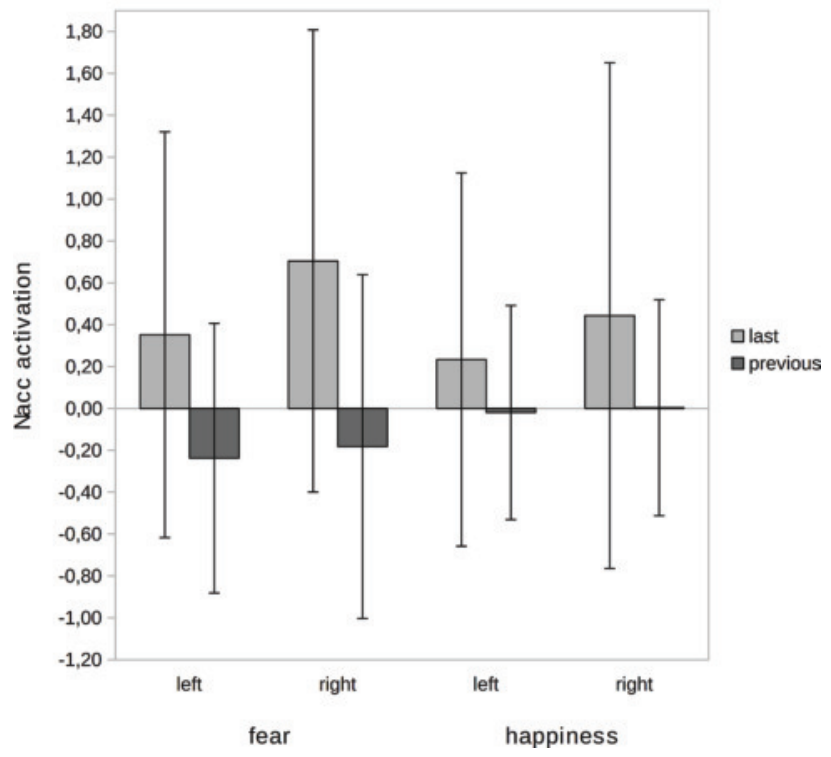

Fig. 4 Interaction between last fearful and last happy face. a Interaction (last fearful face greater than previous fearful faces compared to last happy face greater than previous happy faces), $p<0.001$ uncorrected for display purposes., $k=5,(x, y, z=-9,8,-8)$. b Bars showing mean left and right Nacc activation for each of the conditions. Lines indicate standard deviations with an average number of less than two faces as "L2" and the group with more than two faces as "M2".

Even though L2 looked at fewer faces than M2 [happy: L2: $1.51( \pm 0.35), \mathrm{M} 2: 3.20( \pm 0.82), t(10.2)=8.33$, $p<0.001, d=2.68$. fear: L2: $1.38( \pm 0.39)$, M2: 3.16 ( \pm 0.89$), t(9.9)=7.90, p<0.001, d=2.59$ ], they did not perform significantly worse in the decision on the general emotion within a series [happy: L2: $46.20 \%( \pm 42.49)$, M2: $55.31 \%( \pm 29.65) ; t(45)=0.62, p=0.538, d=0.25$. fear: L2: $51.80 \%( \pm 43.12)$, M2: $61.79 \%( \pm 29.66), t(45)=0.68$, $p=0.501, d=0.27]$. However, comparing contrast estimates of L2 to M2 revealed higher activity during the last fearful face compared to the previous fearful faces in left Nacc $[\mathrm{L} 2: 1.19 \pm 0.55, \mathrm{M} 2: 0.24 \pm 0.36, t(45)=5.22, p<0.001$, $d=2.04$, see Fig. 5], but not for the last happy face in comparison to the previous happy faces [L2: $0.10 \pm 0.55, \mathrm{M} 2$ : $0.03 \pm 0.37, t(45)=0.38, p=0.71, d=0.15]$.

Exploratorily, to allow a correlation approach, instead of using the extreme group, we performed a median split of the whole group. Here, we excluded one outlier, who did not affect the fMRI results across the whole sample, but who drove many of the brain-behavior correlations that were no longer significant after excluding the person. With the median split, 23 persons had looked at less than 2.825 faces per block (L3), and 23 persons had considered more (M3). Analogous to L2 and M2, we refer to the groups as "L3" and "M3".

As illustrated in Fig. 6, the groups showed opposing correlations between Nacc activity for the last compared to all previous faces and the number of faces to reach a decision with a negative correlation in L3 (left: $r=-0.48, p=0.02$, right: $r=-0.40, p=0.06)$ and a tendency for a positive correlation in M3 (left: $r=0.35, p=0.10$; difference of correlation strength to L3: $z=-2.81, p=0.005$, right: $r=0.41$, $p=0.05$, difference of correlation strength to L3: $z=-2.7$, $p=0.003)$.

\section{Correlations of brain activity with questionnaires}

Nacc activation during all last compared to all previous faces was negatively correlated with the number of social roles, assessed with the SNI (left: $r=-0.42, p=0.004$. right: $r=-0.37, p=0.012$ ), with the number of people one is in contact with (left: $r=-0.27, p=0.066$, right: $r=-0.31, p=0.034)$, and with the SPQ constricted affect scale $(r=-0.32 ; p=0.029)$. No other correlations between Nacc activation for all last faces $>$ all previous faces were significant. For the sake of comprehensiveness, it should be mentioned that no significant correlation between Nacc activation and schizotypy occurred when a four factor solution [50], instead of the nine factor solution of the SPQ was applied. 
Table 1 Areas with significant activation for the contrasts of interest at whole-brain level, $p<0.05$ FWE-corrected, $k=5$

\begin{tabular}{|c|c|c|c|c|c|c|c|}
\hline \multirow[t]{2}{*}{ Contrast } & \multirow[t]{2}{*}{ Area } & \multirow[t]{2}{*}{ Brodmann area } & \multirow[t]{2}{*}{ Cluster size $k$} & \multicolumn{3}{|c|}{ MNI-coordinates } & \multirow[t]{2}{*}{$t$ value } \\
\hline & & & & $x$ & $y$ & $z$ & \\
\hline \multirow[t]{3}{*}{ All last $>$ previous } & Putamen left & & 41 & -21 & 14 & -11 & 7.23 \\
\hline & Putamen right & & 45 & 15 & 11 & -11 & 6.74 \\
\hline & Anterior cingulate cortex & BA32 & 28 & 0 & 41 & 16 & 5.21 \\
\hline \multirow[t]{3}{*}{ Fear last $>$ previous } & Anterior cingulate cortex & BA32 & 45 & 6 & 41 & 16 & 6.79 \\
\hline & Putamen left & & 27 & -15 & 8 & -11 & 6.58 \\
\hline & Putamen right & & 21 & 12 & 11 & -8 & 6.09 \\
\hline \multirow[t]{6}{*}{ Fear block } & Visual association cortex, occipital lobe & BA18 & 176 & 12 & -73 & -5 & 10.29 \\
\hline & Pre-motor and supplementary motor cortex, frontal lobe & BA6 & 427 & -21 & -1 & 61 & 8.79 \\
\hline & & & 125 & 27 & -1 & 58 & 8.37 \\
\hline & Somatosensory association cortex, parietal lobe & BA7 & 21 & -9 & -61 & 58 & 6.21 \\
\hline & Inferior frontal gyrus, frontal lobe & BA44 & 5 & -54 & 5 & 19 & 5.89 \\
\hline & Somatosensory association cortex, parietal lobe & BA7 & 8 & 15 & -64 & 58 & 5.75 \\
\hline \multirow[t]{5}{*}{ Happiness block } & Visual association cortex, occipital lobe & BA18 & 157 & 12 & -73 & -5 & 10.24 \\
\hline & Pre-motor and supplementary motor cortex, frontal lobe & BA6 & 109 & 27 & -1 & 58 & 8.29 \\
\hline & & & 304 & -21 & -4 & 58 & 8.22 \\
\hline & Somatosensory association cortex, parietal lobe & BA7 & 10 & -12 & -64 & 55 & 5.76 \\
\hline & & & 8 & 12 & -61 & 58 & 5.58 \\
\hline
\end{tabular}

The comparison of the last happy faces with all previous happy faces revealed no differences in brain activation at the given significance threshold and is thus not listed

Table 2 All significant ROI results for the examined contrasts, $p<0.001, k=5$, peakvoxel $p<0.05 \mathrm{svc}$

\begin{tabular}{|c|c|c|c|c|c|c|c|}
\hline \multirow[t]{2}{*}{ Contrast } & \multirow[t]{2}{*}{ Area } & \multirow[t]{2}{*}{ Hemisphere } & \multirow[t]{2}{*}{ Cluster size $k$} & \multicolumn{3}{|c|}{ MNI-coordinates } & \multirow[t]{2}{*}{$t$ value } \\
\hline & & & & $x$ & $y$ & $z$ & \\
\hline \multirow[t]{2}{*}{ All last $>$ previous } & \multirow[t]{2}{*}{ Nacc } & Left & 75 & -21 & 14 & -11 & 7.23 \\
\hline & & Right & 76 & 15 & 11 & -11 & 6.74 \\
\hline \multirow[t]{2}{*}{ Fear last $>$ previous } & \multirow[t]{2}{*}{ Nacc } & Left & 96 & -15 & 8 & -11 & 6.58 \\
\hline & & Right & 80 & 12 & 11 & -8 & 6.09 \\
\hline \multirow[t]{2}{*}{ Happiness last $>$ previous } & \multirow[t]{2}{*}{ Nacc } & Left & 10 & -21 & 17 & -8 & 3.96 \\
\hline & & Right & 12 & 18 & 14 & -11 & 4.23 \\
\hline \multirow[t]{2}{*}{ Interaction $^{\mathrm{a}}$} & \multirow[t]{2}{*}{ Nacc } & Left & 14 & -9 & 8 & -8 & 3.75 \\
\hline & & Right & 9 & 6 & 8 & -5 & 3.94 \\
\hline \multirow[t]{7}{*}{ Fear block } & \multirow[t]{4}{*}{ BA7 and BA40 } & \multirow[t]{2}{*}{ Left } & 147 & -9 & -61 & 58 & 6.21 \\
\hline & & & 62 & -42 & -34 & 43 & 5.70 \\
\hline & & \multirow[t]{2}{*}{ Right } & 81 & 15 & -64 & 58 & 5.75 \\
\hline & & & 39 & 48 & -31 & 46 & 4.34 \\
\hline & \multirow[t]{3}{*}{ DLPFC } & \multirow[t]{2}{*}{ Left } & 21 & -57 & 5 & 31 & 4.91 \\
\hline & & & 4 & -39 & 32 & 31 & 4.16 \\
\hline & & Right & 14 & 36 & 35 & 34 & 4.37 \\
\hline \multirow[t]{7}{*}{ Happiness block } & \multirow[t]{4}{*}{ BA7 and BA40 } & \multirow[t]{2}{*}{ Left } & 123 & -12 & -64 & 55 & 5.76 \\
\hline & & & 41 & -42 & -34 & 46 & 5.76 \\
\hline & & \multirow[t]{2}{*}{ Right } & 68 & 12 & -61 & 58 & 5.58 \\
\hline & & & 36 & 48 & -31 & 46 & 4.12 \\
\hline & \multirow[t]{3}{*}{ DLPFC } & \multirow[t]{2}{*}{ Left } & 18 & -57 & 8 & 28 & 4.73 \\
\hline & & & 2 & -39 & 32 & 31 & 4.13 \\
\hline & & Right & 9 & 36 & 35 & 34 & 4.14 \\
\hline
\end{tabular}

${ }^{\mathrm{a}}($ Last fearful $>$ previous fearful $)>($ Last happy $>$ previous happy $)$ 


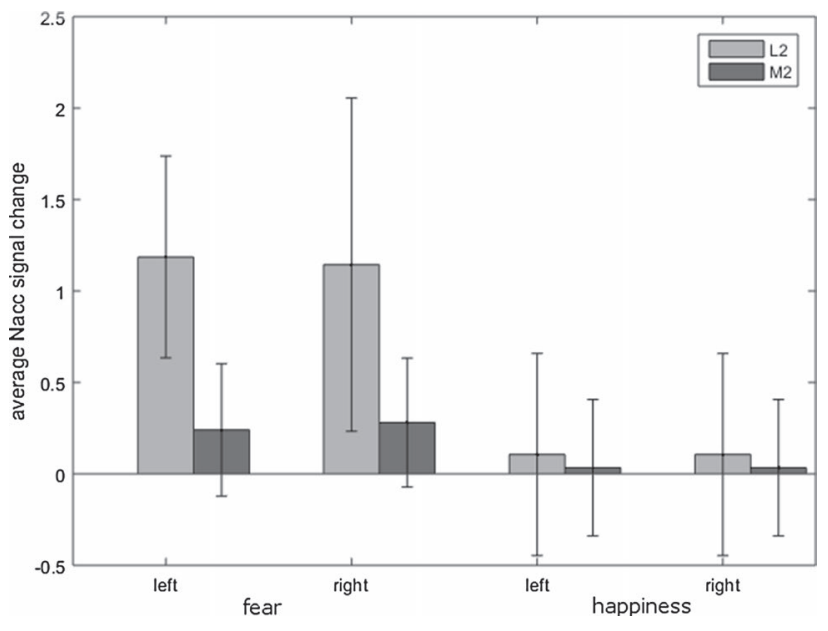

Fig. 5 Bars showing mean left Nacc activation during the last fearful compared to all previous fearful faces and last happy compared to all previous happy faces for the extreme groups L2 $(n=5)$ and M2 $(n=42)$. Lines indicate standard deviations

\section{Discussion}

Our study aimed to investigate the neural correlates of social decision making and its link to (aberrant) salience attribution. Further, we planned on gaining evidence whether salience or reward is the driving factor for Nacc activation during final decision making. To this end, we used an emotion recognition task with morphed pictures, simultaneously expressing fear and happiness to varying degrees.

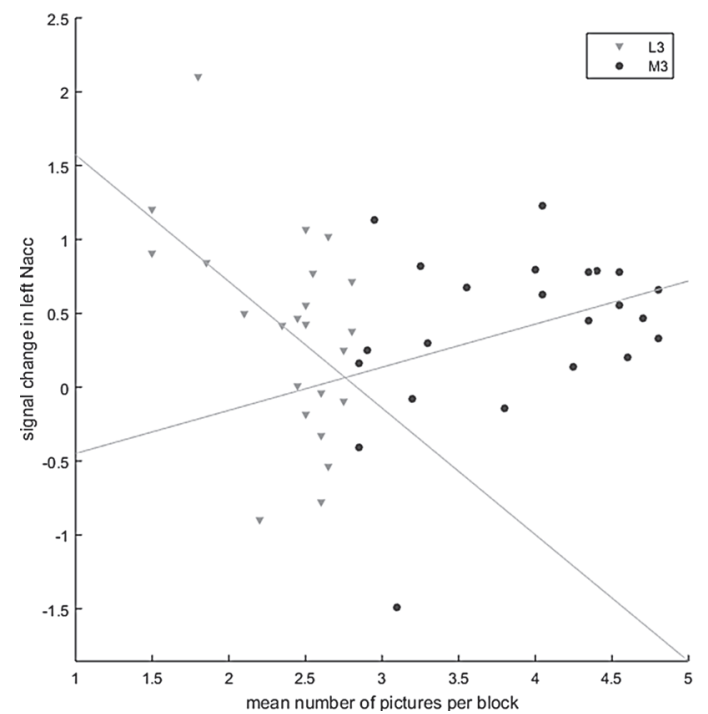

Fig. 6 Correlation of Nacc activation during the last compared to the previous faces with the mean number of faces considered per block. Left: left Nacc. Right: right Nacc. Diamonds: subjects looking at less
Our results successfully replicate the findings from previous studies using non-social stimuli. In line with Esslinger et al. [14], we found activity in the fronto-parietal network during probabilistic reasoning, as well as activity in the Nacc during final decision making. Interestingly, the enhanced activation of the Nacc was accompanied by activation in the anterior cingulate cortex (ACC). The ACC was shown to be involved in reward processing [51], emotional conflict resolution [52], guiding voluntary choices [53], decision making [54], and has a general role in regulating emotional and cognitive processing [55]. Thus in our emotion recognition task, the ACC response might reflect the conflict resolution and according decision for one of the emotions displayed in the morphed facial expressions.

Regarding Nacc activity, we were not only interested in the question whether we can replicate previous findings from JTC-tasks without social stimuli, but also whether reward or salience is the driving factor during final decision making. Esslinger and colleagues [14] concluded from the comparison of rewarded and unrewarded final decisions that salience, but not reward, results in Nacc activity during final decision making, whereas Sabatinelli et al. [12] found activation in Nacc and medial prefrontal cortex to be positively related to pleasantness and reward-value of pictures, but not to unpleasant pictures, salience or arousal. The interaction contrast, i.e., the activation during the last fearful face compared to the previous fearful faces in contrast to the last happy versus previous happy faces, revealed bilaterally enhanced Nacc activation. Hence, in support with the conclusion of Esslinger and colleagues [14], we assume salience, but not reward, to be the driving factor for

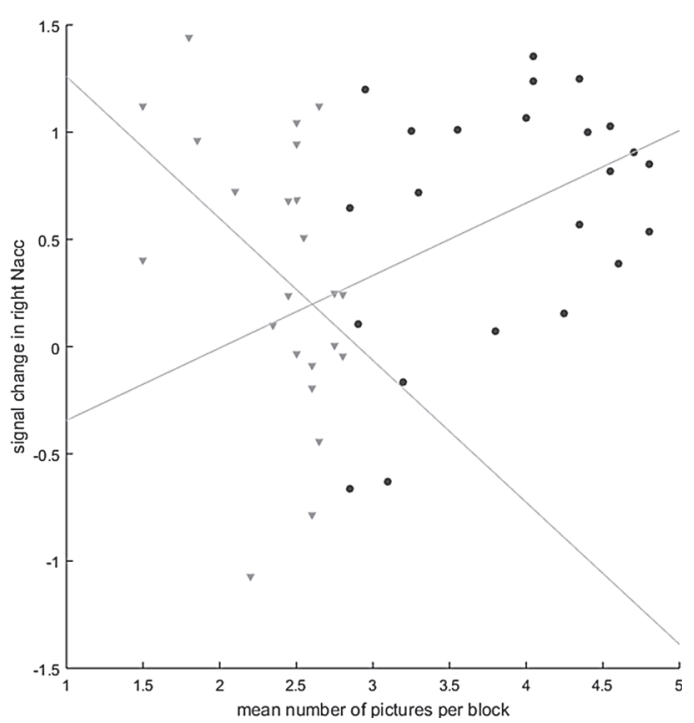

than 2.825 pictures per average block (L3), dots: subjects looking at more than 2.825 faces per average block (M3) 
final decision making. Interestingly, the ACC has also been shown to be involved in salience detection [56], strengthening this interpretation.

As schizophrenia (SZ) is associated with aberrant salience [39, 57], persons with SZ would be expected to have increased Nacc activity during final decision making. Accordingly, healthy participants who showed a JTC-bias in our study indeed had enhanced Nacc activity during final decisions in fearful series. Additionally, analyses of the median-split groups hint toward a possible opposite pattern of Nacc activation between those with and without a JTC-bias tendency, suggesting that fear is more salient to individuals looking at fewer pictures. Further, we revealed a positive association between DTD and performance across participants, linking impaired emotion recognition with hasty decision making. Thus, our results from healthy participants give first evidence that the aberrant salience hypothesis might be extended to explain biased emotion recognition.

Referring to the model of persecutory delusions by Freeman and colleagues [58], it can be assumed that anomalous experiences and arousal are at the heart of the emergence of delusions. The authors define persecutory delusion as a threat belief that results from the interplay of various factors, such as emotions and beliefs, and general cognitive biases with these anomalous experiences / arousal. In our present study we demonstrate a link between aberrant salience (as reflected by enhanced Nacc activation) and a JTC-bias in emotion perception in healthy participants. We assume that a hasty decision about another person's emotion can lead to wrong attributions during emotion recognition. The correlation between DTDs and accuracy supports this assumption, albeit not causally, but only on an associative level. While we are aware of evidence for a negative bias during emotion recognition in SZ [20], but not of evidence for a positive bias, we propose that aberrant salience does not have to lead to biased negative perceptions, but could also result in biased positive perceptions of emotions. Therefore, aberrant salience could result in anomalous experiences and arousal which would influence the interpretation of an emotion as positive or negative, dependent on the own current emotion and general biases. Since negative emotions, and in particular social and general anxiety, play a huge role in SZ [59], the probability for a false-negative perception (i.e., a negative bias) is high, and with this the vulnerability for delusions with negative content, such as persecutory delusions, is increased. Thus, further studies are needed to show whether aberrant salience underlies the specific form of biased emotion recognition that occurs as negative bias for neutral facial expressions in schizophrenia [20], or causes a general emotion bias.

Persons with SZ are known to have reduced social networks [48]. In agreement, correlational analyses with the questionnaires revealed a negative association between the diversity of social roles (spouse, neighbor, close friend, child, coworker, etc.), as well as the network size of individuals and their Nacc activation during the final decision. The additional negative association with constricted affect, as assessed with the SPQ, however, seems at first glance contradictory. Constricted affect belongs to the negative syndrome of schizotypy, and researchers assume opposing effects of negative and positive pathology on social cognition [60]. Importantly, aberrant salience attribution and enhanced Nacc activity is linked to positive pathology, and in particular delusions [39], while negative pathology has been found to go along with reduced Nacc activity [61]. Since a reduced network size might also reflect social withdrawal and negative pathology, the correlational results should be interpreted carefully and warrant replication, especially, because the reported correlations are not corrected for multiple testing.

Several further questions should be addressed in future studies. We found differential activation of the amygdala neither for all last in comparison to all previous faces, nor for the last fearful faces in comparison to the last happy faces, suggesting a reduced role of the amygdala for final decision making. However, since the amygdala is an important brain region for emotion recognition [24, 62] and has been shown to be involved in salience processing [28], further studies are needed to investigate the specific role of the amygdala in social decision making. In addition, to investigate the importance of different brain regions in social decision making more comprehensively, the presented social JTC-task might be analyzed with regard to the right temporoparietal junction and particularly its functional connectivity with the left hippocampus, which recently has been shown to be important for social decision making and social learning in the context of an iterated prisoner's dilemma game [63].

To learn more about brain activation and networks involved in and relevant to the task, it is a necessary next step to invite persons with diagnosed schizotypy or SZ to complete the task; also, inviting healthy participants depending on their self-reported positive schizotypy symptoms, as well as comparing SZ patients with and without delusions would be of high interest. It is noteworthy that patients with SZ did not show Nacc hyperactivation in earlier studies with a non-social JTC-task, but Nacc hypoactivation [21]. If patients also respond with reduced Nacc activation in the social JTC-task, it would be intriguing to find the tipping point in the course of the disease, or within the SZ spectrum, which separates increased from reduced Nacc activation and associated behavioral measures. However, since in our earlier studies we found hypoactivation in our non-social JTC-task, not only for patients with SZ [21], but also for individuals in an at-risk-mental state [10], it can be rather assumed that the pattern of hypo- versus hyperactivity is 
stable across the course of the disease. This is an especially interesting and indeed controversial finding, because in the dopamine hypothesis of SZ, aberrant salience is clearly linked to enhanced subcortical dopamine responding and hyperfunctioning of the Nacc [15, 64]. In agreement with an integrative framework of dopamine functioning for SZ [64], one explanation could be that positive pathology and in particular delusions are characterized by aberrant salience in the form of hypersalience and enhanced Nacc activation which would be linked to hasty decision making. On the contrary, aberrant salience in the form of hyposalience and diminished Nacc responding could be linked to slow decision making and negative pathology, such as apathy. Further, it should be mentioned that Nacc hypoactivity has not only been found during final decision making in SZ [21], but is also a highly stable finding for reward anticipation in SZ that has been linked to deficient salience processing [49, 65], reduced prediction error [66], and the intake of typical antipsychotics [67]. Thus, our findings from the healthy sample are in agreement with predictions of the dopamine hypothesis and the theoretical framework of hasty decision making and hypersalience in SZ [15-17], while the findings of previous studies with SZ patients are not. Future studies should examine whether the proposed association between hasty decision making and delusions that has been confirmed on the behavioral level [16] is also evident in studies investigating Nacc activation during final decision making for social stimuli in SZ.

A limitation is that we did not include a non-social control task, so we cannot directly compare social and nonsocial probabilistic decision making. Future studies including patients with SZ should test both, social and non-social decision making to examine the possibility of divergent activation patterns. Further, based on the observation that antipsychotic medication fails to normalize social cognition and emotion recognition abilities in patients [68], it would be interesting to compare brain activity during the task in medicated versus non-medicated patients. This might provide new insights into the specific effects of the medication with respect to social cognition, and hint towards requirements for drug improvement. In addition, future studies with a focus on the association between delusions and hasty social decision making, might use the emotions happiness and anger, instead of happiness and fear, because anger might be more suitable to cover the perceived threat in paranoid psychosis. A further possible drawback is the usage of stimuli displaying disgust or anger and happiness for the practice trials. We aimed to avoid presenting stimuli that are used in the experiment. This, however, might have led to higher salience for fear than happiness. Still, this neither explains the enhanced activation for the last versus previous fear faces, nor the interaction effect with the emotion (since lower salience should not only occur for the last happy face, but also for all previous happy faces for which we controlled when comparing the last fear with the last happy face). Finally, there was a large variability in block lengths within and between subjects, lasting from almost $20 \mathrm{~s}$ to over $70 \mathrm{~s}$ depending on the number of stimuli considered before deciding on the general emotion. Also, as the block number was fixed, the duration of the experiment depended on the number of stimuli considered. However, participants did not know they could influence the duration of the experiment with their choices. Thus, the measured Nacc signal in the group with a JTCbias might be more noisy (due to less trials for averaging the response to the previous faces, or due to inferior model fit), but should not reflect aberrations in task motivation. In addition, there is evidence suggesting that activation in the Nacc is positively linked with the willingness for task effort $[69,70]$ which is in disagreement with the assumption of reduced motivation causing the higher activation in Nacc and ACC in response to the last face in comparison to all previous faces. Still, we cannot rule out the possibility of reduced motivation influencing the perseverance during each block, and therefore block- and task-length, as well as brain activation.

\section{Conclusions}

We presented results from a social JTC paradigm that allows investigating the neural correlates of social decision making. We show for final decisions during emotion recognition that the Nacc (a) together with the ACC shows strong differential activation, (b) has higher activity in fear than in happiness series, and (c) has higher activity in fear series in participants with a JTC-bias. Based on this first evidence from healthy participants, we suggest that the aberrant salience hypothesis of schizophrenia may be extended to explain biased social cognition. Future studies focusing on the impact of dopamine and salience attribution on social cognition in schizophrenia are highly warranted.

Acknowledgements This research did not receive any specific grant from funding agencies in the public, commercial, or not-for-profit sectors, but was supported by the Heidelberg Academy of Sciences and Humanities. We thank Vera Eymann, Manuel Vietze and Sabine Weinschütz for their help with data acquisition.

Funding The authors received no financial support for the research, authorship, and/or publication of this article.

\section{Compliance with ethical standards}

Conflict of interest The authors declared no potential conflicts of interest with respect to the research, authorship, and/or publication of this article. 


\section{References}

1. Kohler CG, Turner TH, Bilker WB, Brensinger CM, Siegel SJ, Kanes SJ, Gur RE, Gur RC (2003) Facial emotion recognition in schizophrenia: intensity effects and error pattern. Am J Psychiatry 160:1768-1774

2. Kohler CG, Walker JB, Martin EA, Healey KM, Moberg PJ (2010) Facial emotion perception in schizophrenia: a meta-analytic review. Schizophr Bull 36:1009-1019

3. Heerey EA, Bell-Warren KR, Gold JM (2008) Decision-making impairments in the context of intact reward sensitivity in schizophrenia. Biol Psychiatry 64:62-69

4. Moritz S, Woodward TS (2005) Jumping to conclusions in delusional and non-delusional schizophrenic patients. Br J Clin Psychol 44:193-207

5. Howes OD, Kapur S (2009) The dopamine hypothesis of schizophrenia: version III-the final common pathway. Schizophr Bull 35:549-562

6. Matthews SC, Simmons AN, Lane SD, Paulus MP (2004) Selective activation of the nucleus accumbens during risk-taking decision making. Neuroreport 15:2123-2127

7. St Onge JR, Ahn S, Phillips AG, Floresco SB (2012) Dynamic fluctuations in dopamine efflux in the prefrontal cortex and nucleus accumbens during risk-based decision making. J Neurosci 32:16880-16891

8. Zalocusky KA, Ramakrishnan C, Lerner TN, Davidson TJ, Knutson B, Deisseroth K (2016) Nucleus accumbens D2R cells signal prior outcomes and control risky decision-making. Nature 531:642-646

9. Philiastides MG, Auksztulewicz R, Heekeren HR, Blankenburg $\mathrm{F}$ (2011) Causal role of dorsolateral prefrontal cortex in human perceptual decision making. Curr Biol 21:980-983

10. Rausch F, Mier D, Eifler S, Fenske S, Schirmbeck F, Englisch S, Schilling C, Meyer-Lindenberg A, Kirsch P, Zink M (2015) Reduced activation in the ventral striatum during probabilistic decision-making in patients in an at-risk mental state. J Psychiatry Neurosci 40:163-173

11. Kringelbach ML, Berridge KC (2010) The functional neuroanatomy of pleasure and happiness. Discov Med 9:579-587

12. Sabatinelli D, Bradley MM, Lang PJ, Costa VD, Versace F (2007) Pleasure rather than salience activates human nucleus accumbens and medial prefrontal cortex. J Neurophysiol 98:1374-1379

13. Berridge KC (2007) The debate over dopamine's role in reward: the case for incentive salience. Psychopharmacology 191:391-431

14. Esslinger C, Braun U, Schirmbeck F, Santos A, Meyer-Lindenberg A, Zink M, Kirsch P (2013) Activation of midbrain and ventral striatal regions implicates salience processing during a modified beads task. PLoS One 8:e58536

15. Kapur S, Mizrahi R, Li M (2005) From dopamine to salience to psychosis-linking biology, pharmacology and phenomenology of psychosis. Schizophr Res 79:59-68

16. Dudley R, Taylor P, Wickham S, Hutton P. Psychosis (2016) Delusions and the "jumping to conclusions" reasoning bias: a systematic review and meta-analysis. Schizophr Bull 42:652-665

17. Speechley WJ, Whitman JC, Woodward TS (2010) The contribution of hypersalience to the "jumping to conclusions" bias associated with delusions in schizophrenia. J Psychiatry Neurosci 35:7-17

18. Blackwood NJ, Howard RJ, Bentall RP, Murray RM (2001) Cognitive neuropsychiatric models of persecutory delusions. Am J Psychiatry 158:527-539

19. Mier D, Kirsch P (2017) Social-cognitive deficits in schizophrenia. Curr Top Behav Neurosci 30:397-409

20. Mier D, Lis S, Zygrodnik K, Sauer C, Ulferts J, Gallhofer B, Kirsch P (2014) Evidence for altered amygdala activation in schizophrenia in an adaptive emotion recognition task. Psychiatry Res 221:195-203

21. Rausch F, Mier D, Eifler S, Esslinger C, Schilling C, Schirmbeck F, Englisch S, Meyer-Lindenberg A, Kirsch P, Zink M (2014) Reduced activation in ventral striatum and ventral tegmental area during probabilistic decision-making in schizophrenia. Schizophr Res 156:143-149

22. Juckel G, Schlagenhauf F, Koslowski M, Wustenberg T, Villringer A, Knutson B, Wrase J, Heinz A (2006) Dysfunction of ventral striatal reward prediction in schizophrenia. Neuroimage 29:409-416

23. Morris RW, Vercammen A, Lenroot R, Moore L, Langton JM, Short B, Kulkarni J, Curtis J, O'Donnell M, Weickert CS, Weickert TW (2012) Disambiguating ventral striatum fMRIrelated BOLD signal during reward prediction in schizophrenia. Mol Psychiatry 17:235 (280-239)

24. Sergerie K, Chochol C, Armony JL (2008) The role of the amygdala in emotional processing: a quantitative meta-analysis of functional neuroimaging studies. Neurosci Biobehav Rev $32: 811-830$

25. Ohman A (2005) The role of the amygdala in human fear: automatic detection of threat. Psychoneuroendocrinology 30:953-958

26. Hariri AR, Tessitore A, Mattay VS, Fera F, Weinberger DR (2002) The amygdala response to emotional stimuli: a comparison of faces and scenes. Neuroimage 17:317-323

27. Glascher J, Tuscher O, Weiller C, Buchel C (2004) Elevated responses to constant facial emotions in different faces in the human amygdala: an fMRI study of facial identity and expression. BMC Neurosci 5:45

28. Santos A, Mier D, Kirsch P, Meyer-Lindenberg A (2011) Evidence for a general face salience signal in human amygdala. Neuroimage 54:3111-3116

29. Phan KL, Wager T, Taylor SF, Liberzon I (2002) Functional neuroanatomy of emotion: a meta-analysis of emotion activation studies in PET and fMRI. Neuroimage 16:331-348

30. Izuma K, Saito DN, Sadato N (2008) Processing of social and monetary rewards in the human striatum. Neuron 58:284-294

31. Spreckelmeyer KN, Krach S, Kohls G, Rademacher L, Irmak A, Konrad K, Kircher T, Gründer G (2009) Anticipation of monetary and social reward differently activates mesolimbic brain structures in men and women. Soc Cogn Affect Neurosci 2009:nsn051

32. Aharon I, Etcoff N, Ariely D, Chabris CF, O'Connor E, Breiter HC (2001) Beautiful faces have variable reward value: fMRI and behavioral evidence. Neuron 32:537-551

33. Hahn AC, Perrett DI (2014) Neural and behavioral responses to attractiveness in adult and infant faces. Neurosci Biobehav Rev 46(Pt 4):591-603

34. Bishop SJ, Duncan J, Lawrence AD (2004) State anxiety modulation of the amygdala response to unattended threat-related stimuli. J Neurosci 24:10364-10368

35. Anderson IM, Del-Ben CM, McKie S, Richardson P, Williams SR, Elliott R, Deakin JF (2007) Citalopram modulation of neuronal responses to aversive face emotions: a functional MRI study. Neuroreport 18:1351-1355

36. LoBue V (2009) More than just another face in the crowd: superior detection of threatening facial expressions in children and adults. Dev Sci 12:305-313

37. Pinkham AE, Griffin M, Baron R, Sasson NJ, Gur RC (2010) The face in the crowd effect: anger superiority when using real faces and multiple identities. Emotion 10:141-146

38. Jackson ME, Moghaddam B (2001) Amygdala regulation of nucleus accumbens dopamine output is governed by the prefrontal cortex. J Neurosci 21:676-681

39. Kapur S (2003) Psychosis as a state of aberrant salience: a framework linking biology, phenomenology, and pharmacology in schizophrenia. Am J Psychiatry 160:13-23 
40. Savla GN, Vella L, Armstrong CC, Penn DL, Twamley EW (2012) Deficits in domains of social cognition in schizophrenia: a meta-analysis of the empirical evidence. Schizophrenia Bull 39:979-992

41. Huq SF, Garety PA, Hemsley DR (1988) Probabilistic judgements in deluded and non-deluded subjects. Q J Exp Psychol A 40:801-812

42. Woodward TS, Munz M, LeClerc C, Lecomte T (2009) Change in delusions is associated with change in "jumping to conclusions". Psychiatry Res 170:124-127

43. Tottenham N, Tanaka JW, Leon AC, McCarry T, Nurse M, Hare TA, Marcus DJ, Westerlund A, Casey BJ, Nelson C (2009) The NimStim set of facial expressions: judgments from untrained research participants. Psychiatry Res 168:242-249

44. Matzke B, Herpertz SC, Berger C, Fleischer M, Domes G (2014) Facial reactions during emotion recognition in borderline personality disorder: a facial electromyography study. Psychopathology 47:101-110

45. Raine A (1991) The SPQ: a scale for the assessment of schizotypal personality based on DSM-III-R criteria. Schizophr Bull 17:555-564

46. Cohen S, Doyle WJ, Skoner DP, Rabin BS, Gwaltney JM (1997) Social ties and susceptibility to the common cold. JAMA 277:1940-1944

47. Ettinger U, Mohr C, Gooding DC, Cohen AS, Rapp A, Haenschel C, Park S (2015) Cognition and brain function in schizotypy: a selective review. Schizophr Bull 41(Suppl 2):S417-S426

48. Gayer-Anderson C, Morgan C (2013) Social networks, support and early psychosis: a systematic review. Epidemiol Psychiatr Sci 22:131-146

49. Esslinger C, Englisch S, Inta D, Rausch F, Schirmbeck F, Mier D, Kirsch P, Meyer-Lindenberg A, Zink M (2012) Ventral striatal activation during attribution of stimulus saliency and reward anticipation is correlated in unmedicated first episode schizophrenia patients. Schizophr Res 140:114-121

50. Stefanis NC, Smyrnis N, Avramopoulos D, Evdokimidis I, Ntzoufras I, Stefanis CN (2004) Factorial composition of self-rated schizotypal traits among young males undergoing military training. Schizophr Bull 30:335-350

51. Kirsch P, Schienle A, Stark R, Sammer G, Blecker C, Walter B, Ott U, Burkart J, Vaitl D (2003) Anticipation of reward in a nonaversive differential conditioning paradigm and the brain reward system: an event-related fMRI study. Neuroimage 20:1086-1095

52. Etkin A, Egner T, Peraza DM, Kandel ER, Hirsch J (2006) Resolving emotional conflict: a role for the rostral anterior cingulate cortex in modulating activity in the amygdala. Neuron 51:871-882

53. Kennerley SW, Walton ME, Behrens TEJ, Buckley MJ, Rushworth MFS (2006) Optimal decision making and the anterior cingulate cortex. Nat Neurosci 9:940-947

54. Rogers RD, Ramnani N, Mackay C, Wilson JL, Jezzard P, Carter CS, Smith SM (2004) Distinct portions of anterior cingulate cortex and medial prefrontal cortex are activated by reward processing in separable phases of decision-making cognition. Biol Psychiatry 55:594-602

55. Bush G, Luu P, Posner MI (2000) Cognitive and emotional influences in anterior cingulate cortex. Trends Cogn Sci 4:215-222

56. Davis KD, Taylor KS, Hutchison WD, Dostrovsky JO, McAndrews MP, Richter EO, Lozano AM (2005) Human anterior cingulate cortex neurons encode cognitive and emotional demands. J Neurosci 25:8402-8406

57. Grace AA (1991) Phasic versus tonic dopamine release and the modulation of dopamine system responsivity: a hypothesis for the etiology of schizophrenia. Neuroscience 41:1-24

58. Freeman D, Garety PA, Kuipers E, Fowler D, Bebbington PE (2002) A cognitive model of persecutory delusions. Br J Clin Psychol 41:331-347

59. Achim AM, Maziade M, Raymond É, Olivier D, Mérette C, Roy M-A (2009) How prevalent are anxiety disorders in schizophrenia? A meta-analysis and critical review on a significant association. Schizophr Bull 37:811-821

60. Frith CD, Corcoran R (1996) Exploring 'theory of mind' in people with schizophrenia. Psychol Med 26:521-530

61. Wacker J, Dillon DG, Pizzagalli DA (2009) The role of the nucleus accumbens and rostral anterior cingulate cortex in anhedonia: integration of resting EEG, fMRI, and volumetric techniques. Neuroimage 46:327-337

62. Mier D, Lis S, Neuthe K, Sauer C, Esslinger C, Gallhofer B, Kirsch P (2010) The involvement of emotion recognition in affective theory of mind. Psychophysiology 47:1028-1039

63. Bitsch F, Berger P, Nagels A, Falkenberg I, Straube B (2018) The role of the right temporo-parietal junction in social decisionmaking. Hum Brain Mapp 39:3072-3085

64. Maia TV, Frank MJ (2017) An integrative perspective on the role of dopamine in schizophrenia. Biol Psychiatry 81:52-66

65. Nielsen M, Rostrup E, Wulff S, Bak N, Lublin H, Kapur S, Glenth $\varnothing \mathrm{j}$ B (2012) Alterations of the brain reward system in antipsychotic naive schizophrenia patients. Biol Psychiatry 71:898-905

66. Juckel G, Schlagenhauf F, Koslowski M, Wüstenberg T, Villringer A, Knutson B, Wrase J, Heinz A (2006) Dysfunction of ventral striatal reward prediction in schizophrenia. Neuroimage 29:409-416

67. Kirsch P, Ronshausen S, Mier D, Gallhofer B (2007) The influence of antipsychotic treatment on brain reward system reactivity in schizophrenia patients. Pharmacopsychiatry 40:196-196

68. Kucharska-Pietura K, Mortimer A (2013) Can antipsychotics improve social cognition in patients with schizophrenia? CNS Drugs 27:335-343

69. Green MF, Horan WP, Barch DM, Gold JM (2015) Effort-based decision making: a novel approach for assessing motivation in schizophrenia. Schizophr Bull 41:1035-1044

70. Schmidt L, Lebreton M, Cléry-Melin M-L, Daunizeau J, Pessiglione M (2012) Neural mechanisms underlying motivation of mental versus physical effort. PLoS Biol 10:e1001266 